\title{
A Comparison of the Recursive and FFT-based Reassignment Methods in micro-Doppler Analysis
}

\author{
Karol Abratkiewicz*, Piotr Samczyński \\ Institute of Electronic Systems, \\ Warsaw University of Technology, Warsaw, Poland \\ *k.abratkiewicz@elka.pw.edu.pl
}

\author{
Dominique Fourer ${ }^{\circ}$ \\ IBISC, EA 4526 \\ University of Evry/Paris-Saclay, France \\ ○dominique.fourer@univ-evry.fr
}

\begin{abstract}
A brief comparison of two time-frequency (TF) reassignment methods is provided in this paper. Both techniques use the short-time Fourier transform (STFT), however, they can be formulated and computed differently. The first classical method is based on the fast Fourier transform (FFT), while the second one uses a recursive filter bank which, in turn, can be more efficient due to a lower time delay and a reduced computational complexity. Thanks to the proposed methodology, a real-time computation of the spectrogram and the reassigned spectrogram can be obtained. Hence, the reassignment method allows an almost ideal localization of the micro-Doppler signature components in a TF distribution to be obtained. Both approaches are presented, investigated, and validated using real-life radar signals in the form of micro-Doppler signatures originating from different targets.
\end{abstract}

Index Terms-Time-frequency analysis, micro-Doppler, shorttime Fourier transform (STFT), reassignment, real-time.

\section{INTRODUCTION}

In recent years, micro-Doppler analysis has become one of the fundamental techniques in target recognition and classification [1], [2], [3], [4], [5]. This is the result of a relatively simple analysis of the narrowband signal in the baseband, which allows fast algorithms to be applied. Moreover, targets that reflect radar signals may have individual radar signatures, which in consequence allow for their fast and precise classification. Excellent examples are micro-Doppler signatures of drones [6], people [7] and animals [8]. Typically, micro-Doppler signatures are obtained through TF analysis. A common method for this purpose is the STFT. However, this technique suffers from a limited resolution on the TF distribution resulting from the Heisenberg-Gabor uncertainty principle [9]. Additionally, the resolution depends on the analysis parameters (e.g. the number of frequency bins, analysis window length, etc.). Furthermore, these parameters are related to the signal character which can change in time, thus the micro-Doppler signature may have poor resolution if the initial analysis parameters are badly conditioned.

In the literature, several enhancement techniques of the STFT have been proposed such as reassignment and synchrosqueezing with their respective extensions [9], [10], [11], [12], [13], [14]. In general, thanks to these techniques a strong energy concentration of the signal in the TF plan can be obtained, which allows more accurate estimation and decomposition of multicomponent signals. The synchrosqueezing is a variant of the reassignment method which provides invertible concentrated TF distribution and can be combined with additional processing, e.g. filtering, components extraction, among others. As shown in [15], the reassignment method can be successfully used for micro-Doppler signal analysis and can significantly improves the readability of a spectrogram. However, the usual FFT-based approach can suffer from several limitations and trade-off which exclude this technique from being applied in real-time systems. Recently, an efficient alternative implementation of this method was proposed in [16] where the recursive version of the reassigned and synchrosqueezed STFT was introduced. Hence, this paper proposes to investigate and to compare together the classical FFT-based STFT and the recursive filter-bank-based STFT in terms of results and of computational efficiency, when applied in real-time applications in modern radar systems.

The paper is organized as follows: Section II covers the TF reassignment theory, including the FFT-based and recursive approaches. Numerical results obtained using the real-life radar micro-Doppler signals are presented in Section III. In Section IV, a short discussion is provided that explains the differences in both versions of the technique. The summary and conclusions, as well as future plans, are provided in Section V.

\section{TIME-FREQUENCY REASSIGNMENT}

\section{A. Principle}

The reassignment method is a sharpening technique which was first introduced by Kodera et al. [9] to improve the readability of $\mathrm{TF}$ representations. It can be viewed as a post-processing operation which moves the values of the considered TF distribution to new coordinates according to $(t, \omega) \mapsto(\hat{t}(t, \omega), \hat{\omega}(t, \omega))$, where $\hat{t}$ and $\hat{\omega}$ are expected to be closer to the true support of the analyzed signal. In [10], Auger and Flandrin generalized this method to any TF distribution belonging to the Cohen class, such as the spectrogram that is computed from the STFT.

\section{B. FFT-based method}

The STFT provides a function of time $t$ and of frequency $\omega=2 \pi f$ of a signal $x$ using a differentiable analysis window 


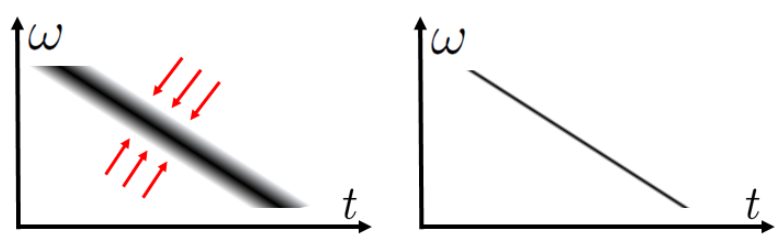

Fig. 1: Graphical illustration of the TF reassignment technique. The left-hand image presents a TF distribution (e.g. a spectrogram) of the linear chirp, the red arrows denote the reassignment operators, and the reassigned spectrogram obtained using Eq. (4) is presented on the right.

$h(t)$. It can be defined as:

$F_{x}^{h}(t, \omega)=\int_{-\infty}^{+\infty} x(\tau) h(t-\tau)^{*} e^{-j \omega \tau} \mathrm{d} \tau=M_{x}(t, \omega) e^{j \Phi_{x}^{h}(t, \omega)}$

where $j^{2}=-1$ and $z^{*}$ is the complex conjugate of $z$. This transform allows one to compute the spectrogram of the analysis signal defined as $\left|F_{x}^{h}(t, \omega)\right|^{2}$.

According to [10], the reassignment operator of the spectrogram can be related to the phase $\Phi_{x}^{h}(t, \omega)$ leading to the following expressions of the reassignment operators:

$$
\begin{array}{ll}
\hat{t}(t, \omega)=-\frac{\partial \Phi_{x}^{h}}{\partial \omega}(t, \omega) & =t-\operatorname{Re}\left(\frac{F_{x}^{T h}(t, \omega)}{F_{x}^{h}(t, \omega)}\right), \\
\hat{\omega}(t, \omega)=\omega+\frac{\partial \Phi_{x}^{h}}{\partial t}(t, \omega) & =\omega+\operatorname{Im}\left(\frac{F_{x}^{D h}(t, \omega)}{F_{x}^{h}(t, \omega)}\right)
\end{array}
$$

where $T h(t)=t h(t)$ and $D h(t)=\frac{\mathrm{d} h}{\mathrm{~d} t}(t)$ are modified versions of the original analysis window $h$.

The last step of the reassignment consists in moving the values of the spectrogram to obtain a sharpened representation called the reassigned spectrogram, expressed as:

$R_{x}^{h}(t, \omega)=\iint_{\mathbb{R}^{2}}\left|F_{x}^{h}(t, \omega)\right|^{2} \delta(t-\hat{t}(t, \omega)) \delta(\omega-\hat{\omega}(t, \omega)) \mathrm{d} t \mathrm{~d} \omega$.

where $\delta(t)$ denotes the Dirac distribution. The discretization process based on the rectangle method leads to the following approximation $F_{x}^{h}[n, m] \approx F_{x}^{h}\left(n T_{s}, \frac{2 \pi m}{M T_{s}}\right), T_{s}=\frac{1}{F_{s}}$ being the sampling period, $n \in \mathbb{Z}$ standing for the time indices, and $m \in[-M / 2 ;+M / 2]$ corresponding to the discrete frequency bin. Hence, each vertical slice of the resulting discrete-time STFT $F_{x}^{h}[n, m]$ can be computed efficiently using the FFT algorithm [17].

\section{Recursive method}

The STFT as defined in Eq. (1) can be expressed as a linear convolution product between the analyzed signal $x$ and a complex valued impulse response of a bandpass filter centered on $\omega, g(t, \omega)=h(t) e^{j \omega t}$ :

$$
\begin{aligned}
y_{x}^{g}(t, \omega) & =\int_{-\infty}^{+\infty} g(\tau, \omega) x(t-\tau) \mathrm{d} \tau=\left|y_{x}^{g}(t, \omega)\right| e^{j \Psi_{x}^{g}(t, \omega)} \\
& =\int_{-\infty}^{+\infty} x(\tau) h(t-\tau) e^{j \omega(t-\tau)} \mathrm{d} \tau \\
& =F_{x}^{h}(t, \omega) e^{j \omega t}=M_{x}^{h}(t, \omega) e^{j\left(\Phi_{x}^{h}(t, \omega)+\omega t\right)}
\end{aligned}
$$

Since $M_{x}^{h}(t, \omega)=\left|y_{x}^{g}(t, \omega)\right|$ and $\Phi_{x}^{h}(t, \omega)=\Psi_{x}^{g}(t, \omega)-\omega t$, the reassignment operator can be reworded as [16]:

$$
\begin{array}{ll}
\hat{t}(t, \omega)=t-\frac{\partial \Psi_{x}^{g}}{\partial \omega}(t, \omega) \quad & =t-\operatorname{Re}\left(\frac{y_{x}^{T g}(t, \omega)}{y_{x}^{g}(t, \omega)}\right), \\
\hat{\omega}(t, \omega)=\frac{\partial \Psi_{x}^{g}}{\partial t}(t, \omega) & =\operatorname{Im}\left(\frac{y_{x}^{D g}(t, \omega)}{y_{x}^{g}(t, \omega)}\right)
\end{array}
$$

with $T g(t, \omega)=t g(t, \omega)$ and $D g(t, \omega)=\frac{\partial g}{\partial \omega}(t, \omega)$.

In [16], [18], a specific analysis window is introduced and allows an implementation in terms of a recursive infinite impulse response (IIR) filtering when discretized:

$$
\begin{aligned}
h_{k}(t) & =\frac{t^{k-1}}{T^{k}(k-1) !} e^{-t / T} U(t), \\
g_{k}(t, \omega) & =h_{k}(t) e^{j \omega t}=\frac{t^{k-1}}{T^{k}(k-1) !} e^{p t} U(t)
\end{aligned}
$$

with $p=-\frac{1}{T}+j \omega, k \geq 1$ the filter order, $T$ the time spread of the window and $U(t)$ the Heaviside step function.

Using the impulse invariance method, the z-transform of the filter $g_{k}(t, \omega)$ allows one to compute the filter coefficients as:

$$
G_{k}(z, \omega)=T_{s} \mathscr{L}\left\{g_{k}(t, \omega)\right\}=\frac{\sum_{i=0}^{k-1} b_{i} z^{-i}}{1+\sum_{i=1}^{k} a_{i} z^{-i}},
$$

with the z-transform $\mathscr{Z}\{f(t)\}=\sum_{n=0}^{+\infty} f\left(n T_{s}\right) z^{-n}$, the filter coefficients $a_{i}=A_{k, i}(-\alpha)^{i}, b_{i}=\frac{1}{L^{k}(k-1) !} B_{k-1, k-i-1} \alpha^{i}$ with $\alpha=e^{p T_{s}}, L=T / T_{s} . B_{k, i}=\sum_{j=0}^{i}(-1)^{j} A_{k+1, j}(i+1-$ $j)^{k}$ denotes the Eulerian numbers and $A_{k, i}=\left(\begin{array}{c}k \\ i\end{array}\right)=\frac{k !}{i !(k-i) !}$ the binomial coefficients. Hence, $y_{k}[n, m] \approx y_{x}^{g_{k}}\left(n T_{s}, \frac{2 \pi m}{M T_{s}}\right)$ can be computed from the sampled analyzed signal $x[n]$ by a standard recursive equation:

$$
y_{k}[n, m]=\sum_{i=0}^{k-1} b_{i} x[n-i]-\sum_{i=1}^{k} a_{i} y_{k}[n-i, m] .
$$

The z-transform of the other specific impulse responses can be computed as functions of $G_{k}(z, \omega)$ at different orders $k \geq 1$ as:

$$
\begin{aligned}
T_{s} \mathscr{L}\left\{T g_{k}(t, \omega)\right\} & =k T G_{k+1}(z, \omega) \\
T_{s} \mathscr{L}\left\{D g_{k}(t, \omega)\right\} & =\frac{1}{T} G_{k-1}(z, \omega)+p G_{k}(z, \omega)
\end{aligned}
$$

with $G_{k}(t, \omega)=0, \forall k<1$. The resulting recursive reassigned spectrogram is then provided by using the discrete-time expression of Eq. (4) with $F_{x}^{h}[n, m]=y_{x}^{g}[n, m] e^{-j 2 \pi \frac{m n}{M}}$.

A graphical illustration of the reassignment technique is depicted in Fig. 1 and holds true for both implementations presented in this section. Thanks to the "energy gathering" properties of this method, a significant energy concentration over the TF plane can be achieved. In the next section, real-life radar signals are processed and compared. 


\section{NUMERICAL EXPERIMENTS}

Here, the authors compare the TF distributions provided by the methods presented in Section II when applied on discretized real-life signals.

\section{A. Materials}

The analyzed signals were collected by the frequency modulated continuous wave (FMCW) XY-DemoRad System developed by XY-Sensing Ltd. [15], [19]. Two different targets were recorded using two available radar analog frontends:

- A walking human was recorded using a K-band radar frontend with the carrier frequency $24 \mathrm{GHz}, 1 \mathrm{GHz}$ bandwidth, and $1 \mathrm{kHz}$ sweep repetition rate,

- A flying drone was recorded using an mm-band radar frontend with the carrier frequency $121 \mathrm{GHz}, 2 \mathrm{GHz}$ bandwidth, and $2 \mathrm{kHz}$ sweep repetition rate.

In both cases the beat signal was distributed on the rangetime plane. Then, for each sweep the range cell in which the object was located in the particular moment (given sweep) was determined. The set of single range cells for each sweep formed a signal with a sampling rate $F_{s}$ imposed by the sweep repetition rate $F_{s}=1 \mathrm{kSa} / \mathrm{s}$ and $F_{s}=2 \mathrm{kSa} / \mathrm{s}$ for each case respectively.

A direct signal was extracted from a range cell in which the observed target was located in each particular moment. This waveform constituted the considered micro-Doppler signal, allowing further TF processing to be performed.

\section{B. Implementation details}

In order to show the usability of the proposed techniques, echoes from a walking human and from a drone were processed using both the recursive and FFT-based approaches to compute a spectrogram and a reassigned spectrogram. In the experiment, the FFT-based method uses a Gaussian analysis window parameterized by a time-spread denoted $\sigma$ such as $h(t)=\frac{1}{\sqrt{2 \pi} \sigma} e^{-\frac{t^{2}}{2 \sigma^{2}}}$. The details of the method parameters for each investigated signal are presented in Table I. These parameters were empirically chosen to provide good TF representation for each signal. Matlab codes of these methods can be found as parts of the ASTRES toolbox [21].

Table I: Processing parameters for two considered techniques and two different signals. $H$ - hop size (stride) in samples, $M$ - number of points along frequency axis, $\sigma$ - time-spread of the analysis Gaussian window, $k$ - filter order, $T$ - time spread of the filter.

\begin{tabular}{|c|c|c|c|}
\hline Method & Parameter & Human walk & Drone flight \\
\hline \multirow{2}{*}{ FFT } & $H[\mathrm{Sa}]$ & 1 & 1 \\
\cline { 2 - 4 } BASED & $M$ & 4096 & 4096 \\
\cline { 2 - 4 } & $\sigma$ & $2.5 \cdot 10^{-2}$ & $1.2 \cdot 10^{-2}$ \\
\hline \multirow{2}{*}{ FILTER } & $k$ & 5 & 11 \\
\cline { 2 - 4 } BANK & $T[\mathrm{~s}]$ & $1.2 \cdot 10^{-2}$ & $5 \cdot 10^{-3}$ \\
\cline { 2 - 4 } & $M$ & 4096 & 4096 \\
\hline
\end{tabular}

Additionally, in order to express the concentration capabilities of the reassignment method the Rényi entropy was computed as a classical measure of the TF distribution concentration [22]. For the investigated cases $\alpha=3$. The outcomes for each distribution are listed in Table II.

\section{Results}

Fig. 2 presents consecutive results obtained using the recursive implementation of the spectrogram and the reassigned spectrogram. For the first signal case, in which the observed target was a receding walking human, the micro-Doppler signature contains a strong signal from a torso oscillating around $-250 \mathrm{~Hz}$ as depicted in Fig. 2a. Additionally, the waving limbs produce components in the whole observed frequency space. However, due to the limited time and frequency resolution, a precise decomposition is challenging, but the reassigned spectrogram allowed for the direct extraction of particular signal terms. After the processing, a straightforward determination of the limb movement parameters is possible, which can be used in classification issues. The same applies for the second observed target. Drone propellers produce a typical broadband echo spread in the Doppler frequency domain (see Fig. 2c) which, after concentration, focuses its energy, allowing for the precise estimation of their speed to be carried out (Fig. 2d). Moreover, the velocity of the whole structure can be distinguished in this way. The Rényi entropy indicates, that the concentrated distributions occupy smaller ares on the TF planes compared to the non-concentrated representations of the signal. This can be deduced by analyzing Table II where the reduced entropy of the reassigned method is presented.

Table II: The third order Rényi entropy $E_{R}^{\alpha}(\cdot)$ before and after concentration for the investigated methods for both signals.

\begin{tabular}{|c|c|c|c|}
\cline { 2 - 4 } \multicolumn{1}{c|}{} & Distribution & $\begin{array}{c}E_{R}^{\alpha}(\cdot) \\
\text { Human walk }\end{array}$ & $\begin{array}{c}E_{R}^{\alpha}(\cdot) \\
\text { Drone flight }\end{array}$ \\
\hline FFT & $\left|F_{x}^{h}(t, \omega)\right|^{2}$ & 20.257 & 21.198 \\
\cline { 2 - 4 } BASED & $R_{x}^{h}(t, \omega)$ & 16.456 & 16.8829 \\
\hline FILTER & $\left|y_{x}^{g}(t, \omega)\right|^{2}$ & 20.337 & 21.1693 \\
\cline { 2 - 4 } BANK & $R_{x}^{g}(t, \omega)$ & 16.015 & 17.553 \\
\hline
\end{tabular}

Comparable outcomes are presented in Fig. 3, where the FFT-based method was used. Due to the different nature of the processing pipeline, slightly different attributes in the distribution are provided, however, in both cases the energy concentration is significant and allows clear decomposition and component separation to be performed. The final resolution is directly dependent on the processing parameters and can be freely tailored to the different signals in order to obtain an efficient energy concentration. Also in this case the Rényi entropy was computed in order to compare the concentration capabilities of the methods for the second investigated signal. Similar to the first signal also in this case the difference in the Rényi entropy before and after concentration is about 4 bits. This shows that both methods can be used interchangeably with similar capacities to concentrate energy on the TF plane which was shown for both investigated signals. Thus, the processed signals and the outcomes confirmed the usability of both techniques in the processing of micro-Doppler radar signals. The next section is devoted to the discussion of the two considered techniques from the implementation perspective. 


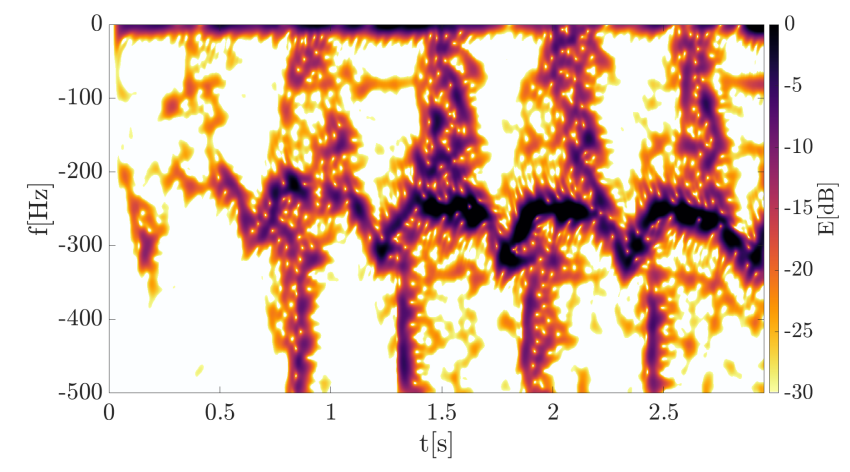

(a) Recursive spectrogram of the echo originating from a walking human

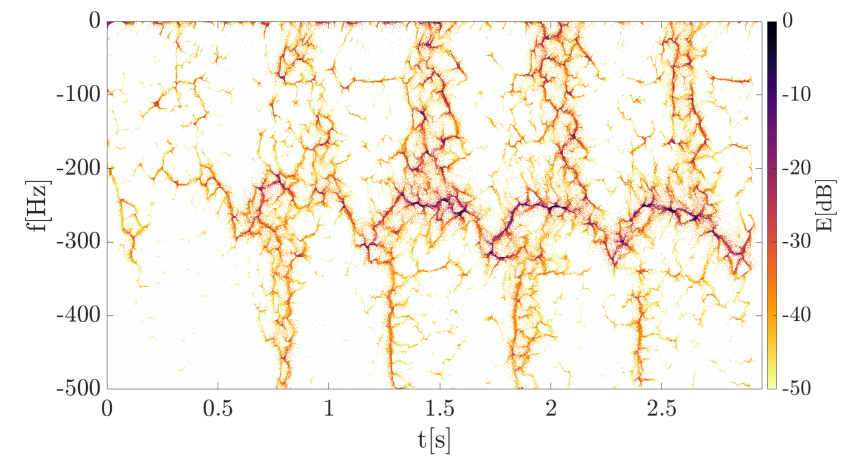

(b) Recursive reassigned spectrogram of the echo originating from a walking human

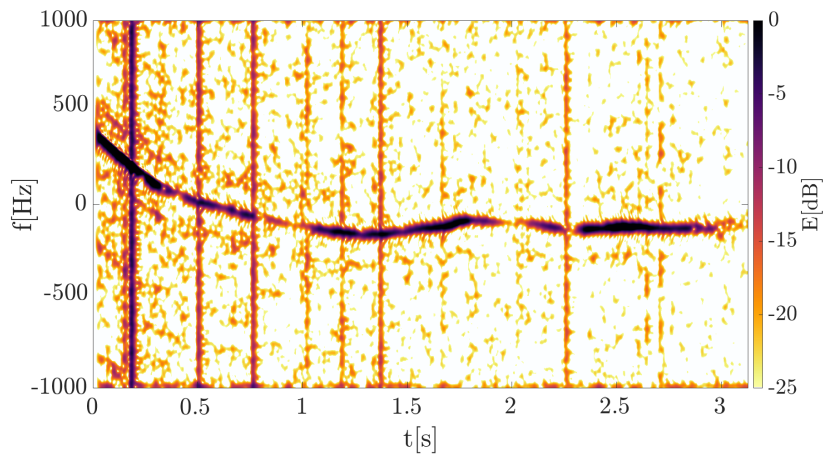

(c) Recursive spectrogram of the echo originating from a drone

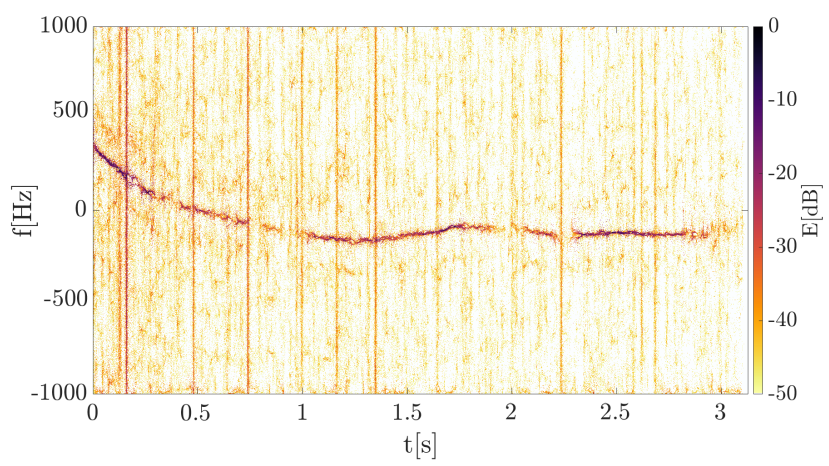

(d) Recursive reassigned spectrogram of the echo originating from a drone

Fig. 2: Results for the recursive method.

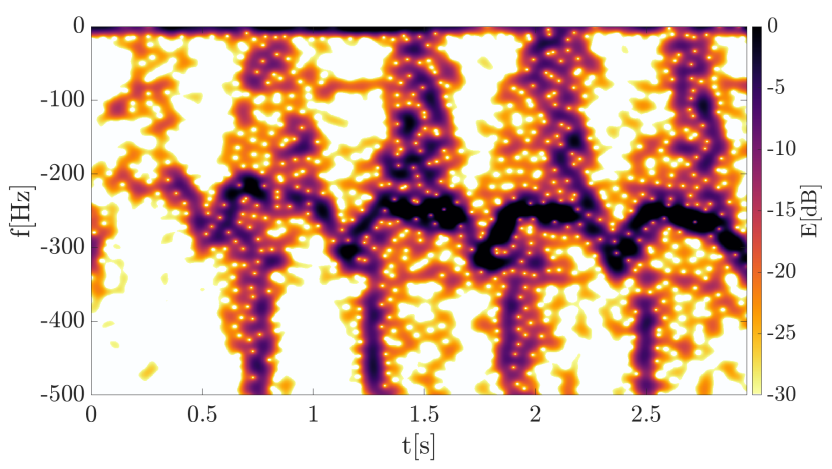

(a) FFT-based spectrogram of the echo originating from a walking human

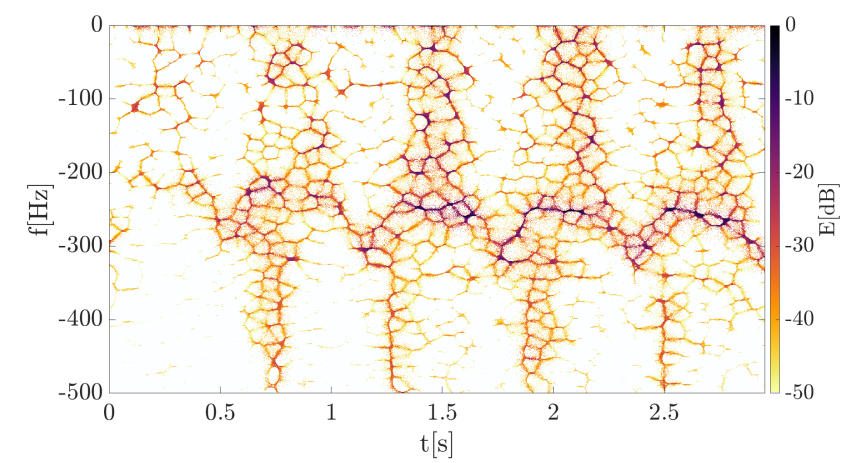

(b) FFT-based reassigned spectrogram of the echo originating from a walking human

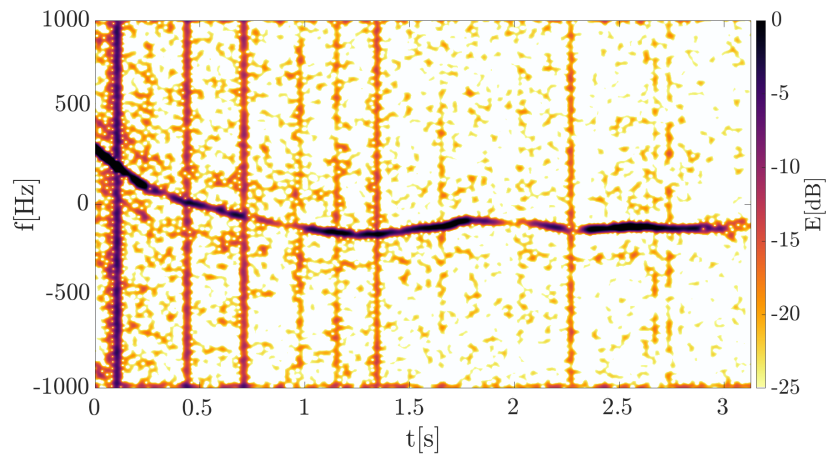

(c) FFT-based spectrogram of the echo originating from a drone

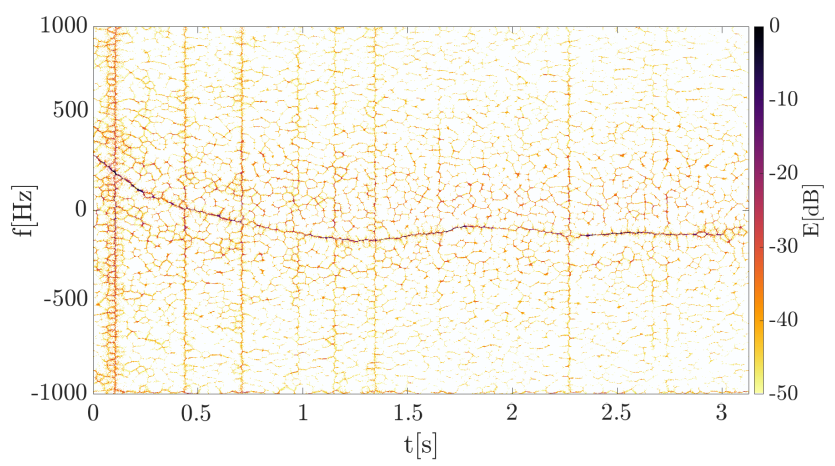

(d) FFT-based reassigned spectrogram of the echo originating from a drone

Fig. 3: Results for the classical FFT-based method. 


\section{COMPARATIVE STUDY}

\section{A. Practical considerations}

As presented in Section III-B, both approaches can provide similar results, hence they can be interchangeably applied in real-life systems. However, the efficiency and simplicity of implementation on embedded platforms promote the recursive filter bank technique. The recursive method has a lower delay proportional to $k$ that is the number of previous signal samples required at each time instant to compute the current STFT coefficients. This number is significantly lower than for the classical FFT-based method which requires at least $M$ samples $(M / 2$ previous samples for a symmetrical window) at each instant to compute a slice of the STFT. Hence, the recursive method allows an implementation on custom off-the shelf processing boards widely available in the market and embedded systems through a IIR filter process which opens a new spectrum of possibilities for this method. Moreover, the filter bank approach offers more adaptability and allows to reduce the computational cost by reducing the number of computed frequency to the region of interest when the bandwidth of the signal is known and limited. As a result, the number of operations through the frequency axis can be lower than $M$ (that is constant for the FFT approach) with the same TF resolution. From another hand, the main advantage of the FFT-based technique is that its precision can be reduced in favor of calculation time. This is typically achieved by increasing the hop size $H$ that corresponds to the number of samples between two successive analyzed windows to compute the STFT. In such a case, decreasing the distribution quality can significantly reduce the computational cost as shown in Fig. 4. The only disadvantages of the recursive technique when compared to the FFT-based approach, are the constrained hop size equal to 1 that cannot be modified by the user, and the constrained causal non-symmetrical analysis window $h_{k}$ which only admits two free user-defined parameters ( $k$ and $T$ ).

\section{B. Computational complexity analysis}

The experiments showed that the recursive approach is less computationally demanding in comparison to the FFT-based method. Indeed, a spectrogram calculation cost expressed in terms of the Landau $\mathcal{O}$ notation for the FFT-based approach leads to $\mathcal{O}\left(N M \log _{2}(M)\right), M$ being the FFT size and $N$ the finite-length of the analyzed signal. From another hand, Eq. (13) allows ones to deduce that $2 k+1$ operations are done to compute each point TF point, leading to the following complexity $\mathcal{G}\left(k N M^{\prime}\right)$ where $M^{\prime} \leq M$ is the number of computed frequency bins. In practice, the FFT-based method can obtain a lower complexity when $H>1$ which results in a poorer time resolution. As a consequence, we have to process a lower number of time samples $N^{\prime}=\lceil N / H\rceil \leq N(\lceil\cdot\rceil$ standing for the ceiling operator) leading to $\mathcal{O}\left(N^{\prime} M \log _{2}(M)\right)$. For both approaches, the reassignment method requires the computation of three STFTs in accordance with Eq. (4): the first one is the STFT with an original window, and the two others ones using respectively the modified windows $D h$ and $T h$. Hence,
Table III: Comparison of the computational complexity between the FFT-based and the reassigned methods.

\begin{tabular}{|ll|}
\hline Method & Run-time complexity in units of time \\
\hline FFT-based STFT & $\mathcal{O}\left(\lceil N / H\rceil M \log _{2}(M)\right)$ \\
recursive STFT & $\mathcal{G}\left(k M^{\prime} N\right)$, with $M^{\prime} \leq M$ \\
\hline FFT-based reassignment & $3 \mathcal{O}\left(\lceil N / H\rceil M \log _{2}(M)\right)$ \\
recursive reassignment & $3 \mathcal{O}\left(k M^{\prime} N\right)$ \\
\hline
\end{tabular}

the computational complexities of the FFT-based and recursive methods are summarized in Table III.

Table IV: Computation time $t_{c}$ for the two investigated signals and the two considered methods.

\begin{tabular}{|c|c|c|c|}
\cline { 2 - 4 } \multicolumn{1}{c|}{} & Distribution & Human walk & Drone flight \\
\hline FFT & $\left|F_{x}^{h}(t, \omega)\right|^{2}$ & $t_{c}=0.105 \mathrm{~s}$ & $t_{c}=0.211 \mathrm{~s}$ \\
\cline { 2 - 4 } BASED & $R_{x}^{h}(t, \omega)$ & $t_{c}=149.641 \mathrm{~s}$ & $t_{c}=436.431 \mathrm{~s}$ \\
\hline FILTER & $\left|y_{x}^{g}(t, \omega)\right|^{2}$ & $t_{c}=1.155 \mathrm{~s}$ & $t_{c}=3.837 \mathrm{~s}$ \\
\cline { 2 - 4 } BANK & $R_{x}^{g}(t, \omega)$ & $t_{c}=181.510 \mathrm{~s}$ & $t_{c}=524.022 \mathrm{~s}$ \\
\hline
\end{tabular}

In Fig. 4, the theoretical computational complexity is presented for each implementation of the reassignment method. The considered case assumed $N=5000 \mathrm{Sa}$, window length $W=128 \mathrm{Sa}$ and three values of the hop size for the FFT-based method: $H_{1}=1, H_{2}=0.25 \cdot \mathrm{W}, H_{3}=0.5 \cdot \mathrm{W}$ and 3 different orders for the recursive method $k=2,5,10$, respectively. The computational complexity is compared for these cases. As

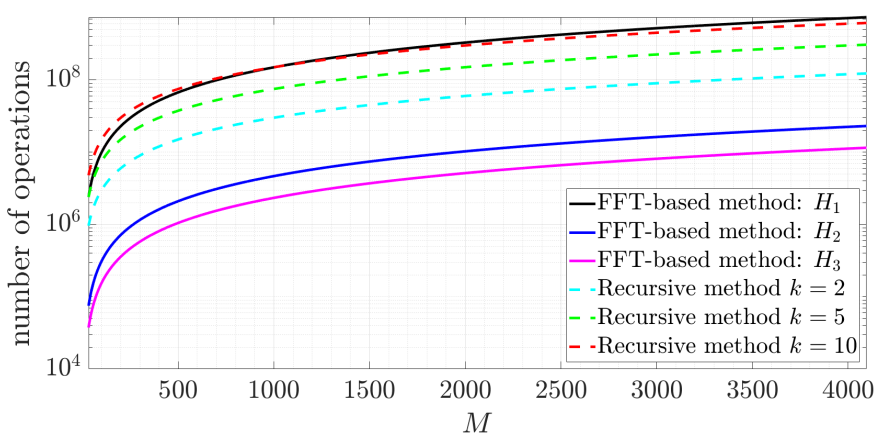

Fig. 4: Comparison of the computational complexity for the considered methods: FFT-based and recursive.

can be observed by analyzing Fig. 4 it is admissible to easy manipulate the parameters to obtain high resolution or reduced processing time. In practical considerations the choice usually comes down to a reasonable compromise allowing sufficient resolution in a sufficiently short time to be achieved. However, precise values depend on the applications, e.g. the processing time may be longer for analyzing walking people, but has to be shorter if fast maneuvering targets are analyzed.

Additionally, the computational time for all investigated methods and signal is listed in Table IV. The processing time is significant for the reassignment method, however, it should be kept in mind, that the computation was performed using demanding parameters (see Table I) which were raised to improve the quality of results. For the purposes of calculations, a computer with an Intel $17-7700 \mathrm{HQ} 2.8 \mathrm{GHz}$ processor, 16 GB DDR4 RAM, an SSD hard drive, and a 64-bit Windows 10 system was used. As can be observed, the processing 
time increase with the filter order which coincides with the theoretical values presented in Table III. In practical real-time applications the processing parameters can be reduced in order to allow fast operation to be performed.

\section{CONCLUSION AND FUTURE WORK}

In this paper, a comparison of two TF reassignment techniques has been presented. Both approaches allow comparable outcomes to be obtained, however, their implementation methodologies are significantly different. The authors paid special attention to the possibilities of implementing the technique on various computing platforms enabling realtime operation. Two real-life micro-Doppler radar signals were processed, which proved the usability of the method and the possibility of their application in real-life systems. The extraction of signal components which resulted from the presented methodology, in the opinion of the authors, should improve the accuracy of classification and allow additional operations on the signal to be performed, such as the filtration of individual components. In the future, the authors intend to develop a real-time implementation of this technique and study the classification techniques, which could be applied to such a TF signal representation.

The presented technique may be applied especially in classification tasks in which the precise signal estimate can increase the system dependability. Following this idea, the micro-Doppler signal is transformed into the $\mathrm{TF}$ plane and then concentrated using the reassignment approach that aims at sharpening the distribution.

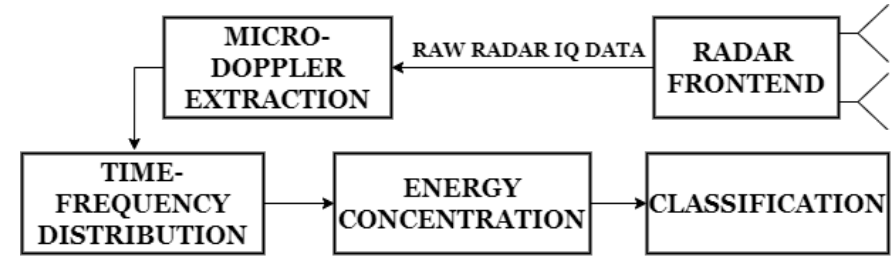

Fig. 5: The idea of the proposed further development of this technique.

As illustrated in Fig. 5, the spectrogram with an improved readability may be used in the classification block. In the authors' opinion, such an approach will increase the accuracy of classification. This can be utilized in security systems, in which the authors' plan for further work. A promising concept is to use a synchrosqueezing method, which instead of relocating the energy along both time and frequency directions, performs energy concentration in one direction [14], [20] to allow signal enhancement and component extraction. This approach is also one of the plans for further research in this matter.

\section{REFERENCES}

[1] V. C. Chen, F. Li, S. -. Ho and H. Wechsler, Micro-Doppler effect in radar: phenomenon, model, and simulation study, in IEEE Trans. on Aerospace and Electronic Systems, vol. 42, no. 1, pp. 2-21, Jan. 2006.

[2] Y. Wang, C. Feng, Y. Zhang and Q. Ge, Classification of Space Targets with Micro-motion Based on Deep CNN, Proc. IEEE 2nd International Conference on Electronic Information and Communication Technology (ICEICT), Harbin, China, 2019, pp. 557-561.
[3] Y. Kim and H. Ling, Human Activity Classification Based on MicroDoppler Signatures Using a SVM, in IEEE Trans. on Geoscience and Remote Sensing, vol. 47, no. 5, pp. 1328-1337, May 2009.

[4] Y. Kim and T. Moon, Human Detection and Activity Classification Based on Micro-Doppler Signatures Using Deep Convolutional Neural Networks, in IEEE Geoscience and Remote Sensing Letters, vol. 13, no. 1, pp. 8-12, Jan. 2016.

[5] A. Huizing, M. Heiligers, B. Dekker, J. de Wit, L. Cifola and R. Harmanny, Deep Learning for Classification of Mini-UAVs Using MicroDoppler Spectrograms in Cognitive Radar, in IEEE Aerospace and Electronic Systems Magazine, vol. 34, no. 11, pp. 46-56, 1 Nov. 2019.

[6] P. Zhang, L. Yang, G. Chen and G. Li, Classification of drones based on micro-Doppler signatures with dual-band radar sensors, Proc. Progress in Electromagnetics Research Symposium - Fall (PIERS - FALL), Singapore, 2017, pp. 638-643.

[7] K. Abratkiewicz, D. Gromek and P. Samczynski, Chirp Rate Estimation and micro-Doppler Signatures for Pedestrian Security Radar Systems, Proc. Signal Processing Symposium (SPSympo), Krakow, Poland, 2019, pp. 212-215.

[8] A. Shrestha et al., Animal Lameness Detection With Radar Sensing, in IEEE Geoscience and Remote Sensing Letters, vol. 15, no. 8, pp. 11891193, Aug. 2018.

[9] K. Kodera, C. de Villedary and R. Gendrin, A new method for the numerical analysis of non-stationary signals, Physics of the Earth and Planetary Interiors, vol. 12, pp. 142-150, 1976.

[10] F. Auger and P. Flandrin, Improving the readability of time-frequency and time-scale representations by the reassignment method, in IEEE Trans. on Signal Processing, vol. 43, no. 5, pp. 1068-1089, May 1995.

[11] S. Meignen, T. Oberlin and S. McLaughlin, A New Algorithm for Multicomponent Signals Analysis Based on SynchroSqueezing: With an Application to Signal Sampling and Denoising, in IEEE Trans. on Signal Processing, vol. 60, no. 11, pp. 5787-5798, Nov. 2012.

[12] F. Auger, E. Chassande-Mottin and P. Flandrin, Making reassignment adjustable: The Levenberg-Marquardt approach, Proc. IEEE International Conference on Acoustics, Speech and Signal Processing (ICASSP), Kyoto, 2012, pp. 3889-3892.

[13] F. Auger et al., Time-Frequency Reassignment and Synchrosqueezing: An Overview, in IEEE Signal Processing Magazine, vol. 30, no. 6, pp. 32-41, Nov. 2013.

[14] D. Fourer, F. Auger, K. Czarnecki, S. Meignen and P. Flandrin, Chirp Rate and Instantaneous Frequency Estimation: Application to Recursive Vertical Synchrosqueezing, in IEEE Signal Processing Letters, vol. 24, no. 11, pp. 1724-1728, Nov. 2017

[15] K. Abratkiewicz, D. Gromek, K. Stasiak and P. Samczyński, TimeFrequency Reassigned Micro-Doppler Signature Analysis Using the XY-DemoRad System, Proc. Signal Processing Symposium (SPSympo), Krakow, Poland, 2019, pp. 331-334.

[16] D. Fourer, F. Auger and P. Flandrin, Recursive versions of the LevenbergMarquardt reassigned spectrogram and of the synchrosqueezed STFT, Proc. IEEE International Conference on Acoustics, Speech and Signal Processing (ICASSP), Shanghai, 2016, pp. 4880-4884.

[17] Henri J. Nussbaumer, The fast Fourier transform. FFT and Convolution Algorithms,. Springer, Berlin, Heidelberg, 1981, pp. 80-111.

[18] G.K. Nilsen, Recursive time-frequency reassignment, IEEE Trans. Signal Process., vol. 57, no. 8, pp. 3283-3287, Aug. 2009.

[19] P. Samczynski, K. Stasiak, D. Gromek, K. Kulpa and J. Misiurewicz, XYDemoRad - Novel K- and mm-Band Radar Demo Kit for Educational and Commercial Applications, 20th International Radar Symposium (IRS), Ulm, Germany, 2019, pp. 1-11.

[20] D. Fourer and F. Auger, Second-order Time-Reassigned Synchrosqueezing Transform: Application to Draupner Wave Analysis, Proc. 27th European Signal Processing Conference (EUSIPCO), A Coruña, Spain, 2019, pp. 1-5.

[21] D. Fourer, J. Harmouche, J. Schmitt, T. Oberlin, S. Meignen, F. Auger and P. Flandrin, The ASTRES toolbox for mode extraction of nonstationary multicomponent signals, Proc. 25th European Signal Processing Conference (EUSIPCO), Kos, Greece, 2017, pp. 1130-1134.

[22] R. G. Baraniuk, P. Flandrin, A. J. E. M. Janssen and O. J. J. Michel, Measuring time-frequency information content using the Renyi entropies, in IEEE Trans. on Information Theory, vol. 47, no. 4, pp. 1391-1409, May 2001. 